[4] R. Fletcher, Practical Methods of Optimization. Chichester, U.K.: Wiley, 1987.

[5] J. C. Geromel, P. L. D. Peres, and J. Bernussou, "On a convex parameter space method for linear control design of uncertain systems," SIAM J. Contr. Optim., vol. 29, no. 2, pp. 381-402, 1991.

[6] E. G. Gilbert and D. W. Johnson, "Distance functions and their application to robot path planning in the presence of obstacles," IEEE Trans. Robotics Automat., vol. RA-1, pp. 21-30, 1985.

[7] E. G. Gilbert and C. Foo, "Computing the distance between general convex objects in three-dimensional space," IEEE Trans. Robotics Automat., vol. 6, pp. 53-61, 1990.

[8] J. E. Hauser, "Proximity algorithms: Theory and implementation," Memorandum no. UCB/ERL M86/53.

[9] K. C. Kiwiel, Methods of Descent for Nondifferentiable Optimization. Berlin, Germany: Springer-Verlag, 1985.

[10] D. G. Northcott, Affine Sets and Affine Groups. Cambridge, U.K.: Cambridge Univ. Press, 1980.

[11] E. Polak, "On the mathematical foundations of nondifferentiable optimization in engineering design," SIAM Rev., vol. 29, pp. 21-89, 1987.

[12] __ Computational Methods in Optimization: A Unified Approach New York: Academic, 1971.

[13] E. Polak and S. E. Salcudean, "On the design of multivariable feedback systems via constrained nondifferentiable optimization in $H^{\infty}$ Saces," IEEE Trans. Automat. Contr., vol. 4, 1989.

[14] Y. H. Zhou, "Proximity algorithms with applications to control," Ph.D. dissertation, Imperial College, Univ. London, 1994

\section{Feedback Limitations in Nonlinear Systems: From Bode Integrals to Cheap Control}

M. M. Seron, J. H. Braslavsky, P. V. Kokotović, and D. Q. Mayne

Abstract-Feedback limitations of nonlinear systems are investigated using the cheap control approach. The main result is that in the limit, when the control effort is free, the smallest achievable $L_{2}$ norm of the output is equal to the least amount of control energy ( $L_{2}$ norm) needed to stabilize the unstable zero dynamics. This nonlinear result is structurally similar to an earlier linear result by Qiu and Davison (1993), which, in turn, is connected with a Bode-type integral derived by Middleton (1991).

Index Terms-Cheap optimal control, nonlinear systems, nonminimum phase systems, performance limitations, zero dynamics.

\section{INTRODUCTION}

In this paper we investigate and quantify obstacles limiting the performance of nonlinear systems. We clarify which structural properties of the nonlinear system prevent any feedback controller from reducing to zero the $L_{2}$ norm of the output.

For linear systems such questions have been answered by classical and modern results, extensively reviewed in a recent monograph [1].

Manuscript received February 2, 1998. Recommended by Associate Editor, L. Qiu. This work was supported in part by the National Science Foundation under Grant ECS-9528370 and the Air Force Office of Scientific Research under Grant F49620-95-1-0409.

M. M. Seron and J. H. Braslavsky are with the Department of Electrical and Computer Engineering, The University of Newcastle, NSW 2308, Australia.

P. V. Kokotović is with the Center for Control Engineering and Computation, University of Calfornia, Santa Barbara, CA 93106 USA.

D. Q. Mayne is with the Department of Electrical and Electronic Engineering, Imperial College, London SW7 2BT U.K.

Publisher Item Identifier S 0018-9286(99)02106-6.
However, most of these linear results are formulated in the frequency domain and are not directly applicable to nonlinear systems.

Feedback limitations of linear systems have also been approached via "cheap" optimal control, which we now extend to nonlinear systems. The key idea is that an optimal controller will be close to its ideal performance if the control effort is cheap and will attain it if the control effort is free. The penalty on the control effort is thus scaled by a small parameter $\varepsilon>0$ and the ideal performance is evaluated in the limit as $\varepsilon \rightarrow 0$.

Although the linear cheap control problem is well understood [2], [3], we revisit it in Section II for two reasons. First, we use it to introduce a singular perturbation technique which we subsequently apply to the nonlinear problem. This parallel development makes the structural similarities of the two problems more apparent. Second, we point out to a heretofore unnoticed connection between Bodetype integrals and linear cheap control. Specifically, we show that a $T$-integral formula derived by Middleton [4] reduces to a limiting cheap control formula derived by Qiu and Davison [3]. The new insight gained is that the value of the $T$-integral is, in fact, the least amount of energy ( $L_{2}$ norm) required to stabilize the unstable zeros of the linear system.

Because of this insight, the nonlinear cheap control analysis in Section III focuses on the unstable zero dynamics - the nonlinear counterpart of nonminimum-phase (NMP) zeros. For simplicity, we assume that the zero dynamics are antistable (asymptotically stable in reverse time) and affinely driven by the output and that the relative degree of the nonlinear system is one. In this way we avoid complicated derivations and gain in clarity. Our main result is that in the limit, as $\varepsilon \rightarrow 0$, the cheap control problem reduces to the minimum energy ( $L_{2}$ norm) problem in which the system output is used to stabilize the nonlinear zero dynamics. This result is a nonlinear counterpart of the Qiu-Davison formula, through which it is also connected with Middleton's integral formula.

\section{FeEdBack Limitations in Linear Systems}

\section{A. Cheap Optimal Control}

The cheap control problem for a stabilizable and detectable linear time-invariant system

$$
\begin{aligned}
& \dot{x}(t)=A x(t)+B u(t), \quad x \in R^{n}, \quad u \in R^{m} \\
& y(t)=C x(t), \quad y \in R^{m}
\end{aligned}
$$

consists of finding a stabilizing state feedback control which minimizes the functional

$$
J_{\varepsilon}=\frac{1}{2} \int_{0}^{\infty}\left[y^{T}(t) y(t)+\varepsilon^{2} u^{T}(t) u(t)\right] d t
$$

when $\varepsilon>0$ is small.

As $\varepsilon \rightarrow 0$, the optimal value $J_{\varepsilon}^{*}$ tends to $J_{0}^{*}$, the ideal performance. Kwakernaak and Sivan proved that $J_{0}^{*}=0$ if and only if the system (1) is minimum phase and right invertible [2, Th. 3.14]. Qiu and Davison derived an explicit formula as a function of the NMP zeros [3]. We will rederive the Qiu-Davison formula under the assumption that the system $(1)$ has relative degree one $(\operatorname{rank} C B=m)$. Then, a change of coordinates $x \mapsto(y, z)$ exists which transforms (1) into the form

$$
\begin{aligned}
& \dot{y}=A_{1} y+A_{2} z+B_{1} u, \quad B_{1}=C B \\
& \dot{z}=B_{0} y+A_{0} z, \quad y \in R^{m}, \quad z \in R^{n-m} .
\end{aligned}
$$


The zeros of $C(s I-A)^{-1} B$ are the eigenvalues of $A_{0}$. We assume that all the zeros are NMP, that is, $-A_{0}$ is Hurwitz.

We now minimize the functional (2) for the system (3). The associated algebraic Riccati equation is

$$
\begin{aligned}
& {\left[\begin{array}{cc}
A_{1} & A_{2} \\
B_{0} & A_{0}
\end{array}\right]^{T} P(\varepsilon)+P(\varepsilon)\left[\begin{array}{ll}
A_{1} & A_{2} \\
B_{0} & A_{0}
\end{array}\right]+\left[\begin{array}{cc}
I & 0 \\
0 & 0
\end{array}\right]} \\
& =\frac{1}{\varepsilon^{2}} P(\varepsilon)\left[\begin{array}{cc}
B_{1} B_{1}^{T} & 0 \\
0 & 0
\end{array}\right] P(\varepsilon) .
\end{aligned}
$$

To find the limit of the positive definite solution $P(\varepsilon)$ as $\varepsilon \rightarrow 0$, we must resolve the singularity at $\varepsilon=0$. Following Jameson and O'Malley [5] we seek $P(\varepsilon)$ in the form of a series in $\varepsilon$

$$
P(\varepsilon)=\left[\begin{array}{cc}
\varepsilon P_{1} & \varepsilon P_{2} \\
\varepsilon P_{2}^{T} & P_{0}+\varepsilon P_{3}
\end{array}\right]+O\left(\varepsilon^{2}\right)
$$

where $P_{0}, P_{1}, P_{2}$, and $P_{3}$ are independent of $\varepsilon$. The substitution of (5) into (4) yields

$$
\begin{aligned}
I-P_{1} B_{1} B_{1}^{T} P_{1}+O(\varepsilon) & =0 \\
B_{0}^{T} P_{0}-P_{1} B_{1} B_{1}^{T} P_{2}+O(\varepsilon) & =0 \\
A_{0}^{T} P_{0}+P_{0} A_{0}-P_{2}^{T} B_{1} B_{1}^{T} P_{2}+O(\varepsilon) & =0 .
\end{aligned}
$$

Setting $\varepsilon=0$, we find $P_{1}=\left(B_{1} B_{1}^{T}\right)^{-1 / 2}$ from (6), and $P_{2}=$ $\left(B_{1} B_{1}^{T}\right)^{-1 / 2} B_{0}^{T} P_{0}$ from (7), and, substituting in (8), we obtain

$$
A_{0}^{T} P_{0}+P_{0} A_{0}=P_{0} B_{0} B_{0}^{T} P_{0} .
$$

Because system (3) is stabilizable and $-A_{0}$ is Hurwitz, the unique positive definite $P_{0}$ exists. By equating to zero the $O(\varepsilon)$ terms (not shown in (6)-(8)) we find $P_{3}=P_{0} B_{0}\left(B_{1} B_{1}^{T}\right)^{-1 / 2} B_{0}^{T} P_{0}$.

Finally, the optimal value $J_{\varepsilon}^{*}=V(y, z ; \varepsilon)$ is calculated to be

$$
\begin{aligned}
J_{\varepsilon}^{*}= & \frac{1}{2} z^{T} P_{0} z+\frac{\varepsilon}{2}\left[y+B_{0}^{T} P_{0} z\right]^{T} \\
& \times\left(B_{1} B_{1}^{T}\right)^{-1}\left[y+B_{0}^{T} P_{0} z\right]+O\left(\varepsilon^{2}\right) \\
= & V_{0}(z)+\varepsilon V_{1}(y, z)+O\left(\varepsilon^{2}\right) .
\end{aligned}
$$

Its limit as $\varepsilon \rightarrow 0$

$$
V_{0}(z)=\frac{1}{2} z^{T} P_{0} z
$$

defines the ideal performance of system (3).

We interpret this result via the minimum energy problem: Find $y$ to stabilize $\dot{z}=A_{0} z+B_{0} y$ and to minimize

$$
J=\frac{1}{2} \int_{0}^{\infty} y^{T}(t) y(t) d t
$$

Here the system output $y$ acts as the control variable for the zero dynamics. The optimal value is $V_{0}(z)$ given in (11). This means that the limiting optimal value for the full system is equal to the least amount of energy required to stabilize the zero-dynamics subsystem.

\section{B. Singular Perturbation Analysis}

When the optimal feedback control

$$
u^{*}=-\frac{1}{\varepsilon} B_{1}^{T}\left(B_{1} B_{1}^{T}\right)^{-1 / 2}\left[y+B_{0}^{T} P_{0} z+O(\varepsilon)\right]
$$

is applied to (3), the resulting closed-loop system is in the standard singular perturbation form

$$
\begin{aligned}
\varepsilon \dot{y} & =-\left(B_{1} B_{1}^{T}\right)^{1 / 2}\left[y+B_{0}^{T} P_{0} z\right]+O(\varepsilon) \\
\dot{z} & =B_{0} y+A_{0} z .
\end{aligned}
$$

For a slow-fast analysis [6] we set $\varepsilon=0$ and get $y=-B_{0}^{T} P_{0} z$. Then, substituting in the $z$-equation, we find that the slow subsystem of (13) is the optimal zero-dynamics subsystem

$$
\dot{z}=-P_{0}^{-1} A_{0}^{T} P_{0} z \text {. }
$$

These slow dynamics evolve in the slow invariant subspace $y+$ $B_{0}^{T} P_{0} z=0$, while the fast dynamics represent the convergence of $y+B_{0}^{T} P_{0} z$ to zero. The two parts of the optimal value are $V_{0}(z)$ slow and $\varepsilon V_{1}(y, z)$ fast. We note that the eigenvalues of (14) are the mirror image of the NMP zeros of (3), a fact known from [2, Th. 3.12].

\section{Cheap Control and the Bode T-Integral}

We now apply the above results to the problem of regulating the output $y$ of (3) to a constant setpoint $r$. With the feedforward term $\bar{u}=-B_{1}^{-1}\left(A_{1}-A_{2} A_{0}^{-1} B_{0}\right) r$ we place the equilibrium of (3) at $y=r$. Introducing the error variables $e=y-r, \tilde{z}=z+A_{0}^{-1} B_{0} r$, and $\tilde{u}=u-\bar{u}$, we rewrite (3) as

$$
\begin{aligned}
& \dot{e}=A_{1} e+A_{2} \tilde{z}+B_{1} \tilde{u} \\
& \dot{\tilde{z}}=A_{0} \tilde{z}+B_{0} e .
\end{aligned}
$$

Then the cheap control problem is the same as for (3), but with $(e, \tilde{z})$ replacing $(y, z)$. The ideal performance is $V_{0}(\tilde{z})$. We are interested in $V_{0}(\tilde{z}(0))$ for the initial condition $e(0)=r, \tilde{z}(0)=A_{0}^{-1} B_{0} r$, which corresponds to the state $y$ being transferred from zero to $r$.

Qiu-Davison Formula: For the initial condition $e(0)=r, \tilde{z}(0)=$ $A_{0}^{-1} B_{0} r$, the ideal performance of (15) is

$$
V_{0}(\tilde{z}(0))=\frac{1}{2} r^{T} H r, \quad \text { where } \quad \text { trace } H=2 \sum_{i=1}^{k} \frac{1}{\alpha_{i}}
$$

and $\alpha_{1}, \cdots, \alpha_{k}$ are the unstable eigenvalues of $A_{0}$, that is the NMP zeros of system (15). This formula is obtained from (11) and (9)

$$
\begin{aligned}
V_{0}(\tilde{z}(0)) & =\frac{1}{2} \tilde{z}^{T}(0) P_{0} \tilde{z}(0) \\
& =\frac{1}{2} r^{T} B_{0}^{T}\left(A_{0}^{T}\right)^{-1} P_{0} A_{0}^{-1} B_{0} r \triangleq \frac{1}{2} r^{T} H r \\
\operatorname{trace} H & =\operatorname{trace}\left[P_{0}^{1 / 2} A_{0}^{-1} B_{0} B_{0}^{T}\left(A_{0}^{T}\right)^{-1} P_{0}^{1 / 2}\right] \\
& =\operatorname{trace}\left[P_{0}^{1 / 2} A_{0}^{-1} P_{0}^{-1 / 2}+P_{0}^{-1 / 2}\left(A_{0}^{T}\right)^{-1} P_{0}^{1 / 2}\right] \\
& =2 \operatorname{trace} A_{0}^{-1}=2 \sum_{i=1}^{k} \frac{1}{\alpha_{i}} .
\end{aligned}
$$

How is this result related to the performance limitations defined by well-known Bode integrals? An integral formula for the complementary sensitivity function $T$ derived by Middleton [4] is

$$
\frac{1}{\pi} \int_{0}^{\infty} \log |T(j \omega)| \frac{d \omega}{\omega^{2}}+\frac{1}{2 K_{v}}=\sum_{i=1}^{k} \frac{1}{\alpha_{i}}
$$

where $K_{v}$ is the velocity constant. The glaring similarity of the Middleton formula (17) and the Qiu-Davison formula (16) has, apparently, remained unnoticed.

Proposition (Cheap Control and Bode T-Integral): Let $G$ and $K$ be the single-input/single-output transfer functions of a plant and a minimum phase controller, respectively. Suppose that $T=G K(1+$ $G K)^{-1}$ is stable and $T(0)=1$. Then

$$
\frac{1}{\pi} \int_{0}^{\infty} \log |T(j \omega)| \frac{d \omega}{\omega^{2}}+\frac{1}{2 K_{v}}=\lim _{\varepsilon \rightarrow 0} \frac{1}{2} \int_{0}^{\infty} e^{2}(t) d t
$$

where $e(t)=y(t)-r$ is the error in transferring the system from rest to the setpoint $r=1$ using the cheap optimal control.

A dual result relates the classical Bode integral for the sensitivity function $S$ [7] with the minimum energy ( $L_{2}$ norm) required to transfer the system to rest from the initial condition originating from a unit impulse at the input [8]. Similar relations have been derived in linear filtering [9]. 


\section{Feedback Limitations in Nonlinear Systems}

\section{A. Cheap Optimal Control}

We now proceed to the main subject of this paper, i.e., the lowest attainable $L_{2}$ norm of the output $y$ of the nonlinear system

$$
\begin{aligned}
& \dot{y}=f(y, z)+g(y, z) u, \quad y, u \in R^{m} \\
& \dot{z}=f_{0}(z)+g_{0}(z) y, \quad z \in R^{n-m}
\end{aligned}
$$

where $f(0,0)=0, f_{0}(0)=0$. The following assumption implies that the relative degree of (18) is one and that its zero dynamics are governed by $\dot{z}=f_{0}(z)$ [10].

Assumption I: There exists $\gamma>0$ such that the smallest singular value of $g(y, z)$ is greater than or equal to $\gamma$ for all $y$ and $z$.

For the nonlinear system (18), the cheap optimal control problem consists of finding a feedback control $u$ which guarantees asymptotic stability and minimizes the functional (2) when $\varepsilon>0$ is small. It is well known (e.g., [11, p. 91]) that this problem has a solution if there exists a positive semidefinite optimal value $V(y, z ; \varepsilon)$ which satisfies the Hamilton-Jacobi-Bellman equation (HJBE)

$$
\begin{aligned}
& \frac{\partial V}{\partial y} f(y, z)+\frac{\partial V}{\partial z}\left[f_{0}(z)+g_{0}(z) y\right]+\frac{1}{2} y^{T} y \\
& \quad-\frac{1}{2 \varepsilon^{2}} \frac{\partial V}{\partial y} g(y, z) g^{T}(y, z) \frac{\partial^{T} V}{\partial y}=0, \quad V(0,0 ; \varepsilon)=0
\end{aligned}
$$

and such that the feedback control

$$
u=-\frac{1}{\varepsilon^{2}} g^{T}(y, z) \frac{\partial^{T} V}{\partial y}
$$

asymptotically stabilizes (18). We are interested in the limit of $V(y, z ; \varepsilon)$ as $\varepsilon \rightarrow 0$. At $\varepsilon=0$ the HJBE (19) has a singularity, which we try to resolve by seeking a solution in the form

$$
V(y, z ; \varepsilon)=V_{0}(z)+\varepsilon V_{1}(y, z)+O\left(\varepsilon^{2}\right) .
$$

Its substitution in (19) yields

$$
\begin{aligned}
& \frac{\partial V_{0}}{\partial z}\left[f_{0}(z)+g_{0}(z) y\right]+\frac{1}{2} y^{T} y-\frac{1}{2} \frac{\partial V_{1}}{\partial y} g(y, z) g^{T}(y, z) \frac{\partial^{T} V_{1}}{\partial y} \\
& \quad+O(\varepsilon)=0 .
\end{aligned}
$$

A closer examination of (22) shows that the first two terms represent the Hamiltonian for the minimum energy problem: Find $y$ to asymptotically stabilize the zero-dynamics subsystem

$$
\dot{z}=f_{0}(z)+g_{0}(z) y
$$

and to minimize the cost (12). We therefore make the following assumption.

Assumption 2: The zero dynamics of (18) are antistable ( $\dot{z}=$ $-f_{0}(z)$ is asymptotically stable), and there exists a positive definite function $V_{0}(z)$ that satisfies the HJBE

$$
\frac{\partial V_{0}}{\partial z} f_{0}(z)-\frac{1}{2} \frac{\partial V_{0}}{\partial z} g_{0}(z) g_{0}^{T}(z) \frac{\partial^{T} V_{0}}{\partial z}=0, \quad V_{0}(0)=0
$$

and such that the feedback control

$$
y=\alpha_{0}(z) \triangleq-g_{0}^{T}(z) \frac{\partial^{T} V_{0}}{\partial z}
$$

achieves global asymptotic stability (GAS) of (23).

With $V_{0}(z)$ so defined, we return to (22), add and subtract $\frac{1}{2}\left\|\alpha_{0}(z)\right\|^{2}=\frac{1}{2}\left\|g_{0}^{T} \partial^{T} V_{0} / \partial z\right\|^{2}$, and, using (24), obtain

$$
\frac{1}{2}\left[y-\alpha_{0}(z)\right]^{T}\left[y-\alpha_{0}(z)\right]-\frac{1}{2} \frac{\partial V_{1}}{\partial y} g(y, z) g^{T}(y, z) \frac{\partial^{T} V_{1}}{\partial y}=O(\varepsilon) .
$$

Defining $\eta=y-\alpha_{0}(z), \tilde{g}(\eta, z)=g\left(\eta+\alpha_{0}(z), z\right)$, and $\tilde{V}_{1}(\eta, z)=$ $V_{1}\left(\eta+\alpha_{0}(z), z\right)$, and letting $\varepsilon=0$, we rewrite (26) as

$$
\frac{1}{2} \eta^{T} \eta-\frac{1}{2} \frac{\partial \tilde{V}_{1}}{\partial \eta} \tilde{g}(\eta, z) \tilde{g}^{T}(\eta, z) \frac{\partial^{T} \tilde{V}_{1}}{\partial \eta}=0 .
$$

This is the HJBE for the cheap control problem: Find $u$ to stabilize the system $\dot{\eta}=\tilde{g}(\eta, z) u$, where $z$ is treated as a constant vector, and to minimize the functional $\frac{1}{2} \int_{0}^{\infty}\left[\eta^{T} \eta+\varepsilon^{2} u^{T} u\right] d t$. The optimal value for this problem is $\varepsilon \tilde{V}_{1}(\eta, z)$ and the optimal control is

$$
u=-\frac{1}{\varepsilon} \tilde{g}^{T} \frac{\partial^{T} \tilde{V}_{1}}{\partial \eta}=-\frac{1}{\varepsilon} \tilde{g}^{T}\left(\tilde{g} \tilde{g}^{T}\right)^{-1 / 2} \eta .
$$

Thus, the original cheap control problem (18), (2) has decomposed in two subproblems: a minimum energy problem for the zerodynamics subsystem (23) and a cheap control problem to rapidly regulate $\eta=y-\alpha_{0}(z)$ to zero.

\section{B. Singular Perturbation Analysis}

We treat (28) as an approximation of the optimal control (20) and apply it to (18). In the $(\eta, z)$-coordinates, the resulting feedback system is

$$
\begin{aligned}
\varepsilon \dot{\eta} & =-\left[\tilde{g}(\eta, z) \tilde{g}^{T}(\eta, z)\right]^{1 / 2} \eta+\varepsilon \phi(\eta, z) \\
\dot{z} & =f_{0}(z)+g_{0}(z) \alpha_{0}(z)+g_{0}(z) \eta
\end{aligned}
$$

where $\phi(\eta, z)=f\left(\eta+\alpha_{0}(z), z\right)-\dot{\alpha}_{0}(z)$.

This system is in standard singular perturbation form [6]. We investigate its stability properties. In the slow manifold $\eta=0$ the system reduces to the slow subsystem

$$
\dot{z}=f_{0}(z)+g_{0}(z) \alpha_{0}(z)
$$

which, by Assumption 2, is GAS. Convergence to the slow manifold is governed by the fast (boundary-layer) subsystem

$$
\frac{d \eta}{d \tau}=-\left[\tilde{g}(\eta, z) \tilde{g}^{T}(\eta, z)\right]^{1 / 2} \eta, \quad \tau=\frac{t}{\varepsilon}
$$

with $z$ treated as a constant vector. The fast subsystem (31) is globally exponentially stable by Assumption 1 .

As shown in [12], these stability properties of the slow and fast subsystems (30) and (31) guarantee semiglobal (in $\varepsilon$ ) boundedness of the solutions of (29). For each pair of positive numbers $(R, \rho)$, there exists $\varepsilon_{R}>0$ such that for all $\varepsilon \in\left(0, \varepsilon_{R}\right]$ the solutions of (29) starting in the ball $B_{R}$ of radius $R$ enter in finite time the ball $B_{\rho}$.

However, this is not sufficient for asymptotic stability of the equilibrium $(\eta, z)=(0,0)$ : the system $\varepsilon \dot{\eta}=-\eta+\varepsilon z, \dot{z}=-z^{3}+\eta$ has slow and fast subsystems as above, but for $\varepsilon>0$ its equilibrium is unstable [12]. As suggested in [13], we need to restrict the interconnection $\phi(\eta, z)$.

Lemma (Asymptotic Stability): Suppose $\|\phi(\eta, z)\| \leq k_{1}\|\eta\|+$ $k_{2}\left\|\alpha_{0}(z)\right\|$ in $B_{\delta}$ for some $\delta>0$. Then, under Assumptions 1 and 2 , to each $R>0$ there corresponds an $\varepsilon_{R}>0$ such that for all $\varepsilon \in\left(0, \varepsilon_{R}\right]$ the equilibrium $(\eta, z)=(0,0)$ of (29) is asymptotically stable and its basin of attraction contains $B_{R}$.

Proof: Using (24) and (25), the derivative of the Lyapunov function $W(\eta, z)=\frac{1}{2} \eta^{T} \eta+V_{0}(z)$ along the solutions of (29) in $B_{\delta}$ satisfies

$$
\dot{W} \leq-\frac{1}{2}\left[\begin{array}{c}
\|\eta\| \\
\left\|\alpha_{0}(z)\right\|
\end{array}\right]^{T}\left[\begin{array}{cc}
2\left(\frac{\gamma}{\varepsilon}-k_{1}\right) & 1+k_{2} \\
1+k_{2} & 1
\end{array}\right]\left[\begin{array}{c}
\|\eta\| \\
\left\|\alpha_{0}(z)\right\|
\end{array}\right]
$$

where $\gamma$ is given by Assumption 1 .

Thus, $\dot{W} \leq 0$ for all $\varepsilon \in\left(0, \varepsilon_{a}\right]$, where $\varepsilon_{a}=\gamma /\left[\left(1+k_{2}\right)^{2}+2 k_{1}\right]$. Pick $c$ such that $\Omega_{c}=\{(\eta, z): W(\eta, z) \leq c\} \subset B_{\delta}$. Then, by LaSalle's invariance theorem, every solution of (29) starting in $\Omega_{c}$ 
converges to the largest invariant set contained in $E=\{(\eta, z)$ : $\dot{W}=0\} \subset\left\{(\eta, z): \eta=0, \alpha_{0}(z)=0\right\}$. Because, by Assumption 2, $y=\alpha_{0}(z)$ asymptotically stabilizes (23), this invariant set is just $(\eta, z)=(0,0)$, which is then asymptotically stable with a basin of attraction that contains $\Omega_{c}$.

Next, choose $\rho>0$ such that $B_{\rho} \subset \Omega_{c}$. Then, [12, Th. 1] guarantees the existence of $\varepsilon_{R} \in\left(0, \varepsilon_{a}\right]$ such that for all $\varepsilon \in\left(0, \varepsilon_{R}\right]$ the solutions of (29) starting in $B_{R}$ enter $B_{\rho}$ in finite time and thereafter converge to $(\eta, z)=(0,0)$.

\section{Limitations to Nonlinear Ideal Performance}

Thus far we have established that $V_{0}+\varepsilon V_{1}$ satisfies the HJBE (19) with an $O(\varepsilon)$ error and that the corresponding control, given by (28) $u=-\frac{1}{\varepsilon^{2}} g^{T} \frac{\partial^{T}\left(V_{0}+\varepsilon V_{1}\right)}{\partial y}=-\frac{1}{\varepsilon} \tilde{g}^{T} \frac{\partial^{T} \tilde{V}_{1}}{\partial \eta}=-\frac{1}{\varepsilon} \tilde{g}^{T}\left(\tilde{g} \tilde{g}^{T}\right) V^{1 / 2} \eta$ asymptotically stabilizes (18), written in closed-loop form as (29).

Our analysis has decomposed the optimal cheap control problem in two separate subproblems: a minimum energy problem for asymptotic stabilization of the zero-dynamics subsystem (23) and a cheap control problem for asymptotic stabilization of the boundary-layer subsystem (31). The solutions of (29), as well as those of (18) and (20), begin by rapidly ("instantaneously" as $\varepsilon \rightarrow 0$ ) converging to the zero-dynamics manifold $\eta=y-\alpha_{0}(z)=0$. After that, they settle in a slow motion during which the zero dynamics are controlled by $y=\alpha_{0}(z)$. The cost of the rapid transient vanishes as $\varepsilon \rightarrow 0$. What remains is the cost $V_{0}(z)$ of the slow motion needed to stabilize the unstable zero dynamics. The overall cost cannot be reduced below $V_{0}(z)$. We are now ready to state and prove our main result.

Theorem (Ideal Performance): Under the conditions of the lemma, for every initial state $(y(0), z(0))$ for which the cheap control problem (18), (2) has a solution, the optimal value satisfies

$$
J_{\varepsilon}^{*}=V(y(0), z(0) ; \varepsilon)=V_{0}(z(0))+O(\varepsilon)
$$

and thus the ideal performance is $V_{0}(z(0))$, the optimal value of the minimum energy problem for the zero-dynamics subsystem (23) controlled by the output $y$.

Proof: We prove that the cost produced by the control (28) satisfies (33). Since the optimal control cannot produce a larger cost, (33) will therefore hold for the optimal value. Let $t_{\delta}$ be the finite time such that $(\eta(t), z(t)) \in B_{\delta}$ for all $t \geq t_{\delta}$. By integrating (32) over $\left[t_{\delta}, \infty\right)$ we obtain

$$
\begin{aligned}
\int_{t_{\delta}}^{\infty} & {\left[\frac{1}{2}\left\|\alpha_{0}(z)\right\|^{2}+\left(1+k_{2}\right)\left\|\alpha_{0}(z)\right\|\|\eta\|+\left(\frac{\gamma}{\varepsilon_{R}}-k_{1}\right)\|\eta\|^{2}\right] d t } \\
\leq & V_{0}\left(z\left(t_{\delta}\right)\right)+\frac{1}{2}\left\|\eta\left(t_{\delta}\right)\right\|^{2}<\infty .
\end{aligned}
$$

This, along with the boundedness of the solutions of (29) on $\left[0, t_{\delta}\right]$, guarantees that the cost

$$
J_{\varepsilon}=\int_{0}^{\infty}\left[\frac{1}{2}\left\|\alpha_{0}(z)\right\|^{2}+\alpha_{0}^{T}(z) \eta+\|\eta\|^{2}\right] d t
$$

is finite. Observing that (24) and (29) yield $\frac{1}{2}\left\|\alpha_{0}(z)\right\|^{2}+\alpha_{0}^{T}(z) \eta=$ $-\dot{V}_{0}$, we express (35) as

$$
J_{\varepsilon}=V_{0}(z(0))+\int_{0}^{\infty}\|\eta\|^{2} d t .
$$

Multiplying the $\eta$ equation in (29) by $\eta^{T}$

$$
\eta^{T} \dot{\eta}=-\frac{1}{\varepsilon} \eta^{T}\left(\tilde{g} \tilde{g}^{T}\right)^{1 / 2} \eta+\eta^{T} \phi \leq-\frac{\gamma}{\varepsilon}\|\eta\|^{2}+\eta^{T} \phi
$$

and integrating we get

$$
\frac{\gamma}{\varepsilon} \int_{0}^{\infty}\|\eta\|^{2} d t \leq \frac{1}{2}\|\eta(0)\|^{2}+\int_{0}^{t_{\varepsilon}} \eta^{T} \phi d t+\int_{t_{\delta}}^{\infty} \eta^{T} \phi d t .
$$

Since, by assumption, the bound $\|\phi\| \leq k_{1}\|\eta\|+k_{2}\left\|\alpha_{0}\right\|$ holds on $\left[t_{\delta}, \infty\right)$, it follows from (34) and (37) that $\int_{0}^{\infty}\|\eta\|^{2} d t$ in (36) is $O(\varepsilon)$, which proves $(33)$.

Thus, the lowest attainable $L_{2}$ norm of the output of (18) is the least amount of energy required to stabilize the unstable zero dynamics. Although essentially nonlinear, this result is structurally similar to its linear counterpart.

It is also of interest to find a nonlinear analog of the property that, as $\varepsilon \rightarrow 0$, the finite poles of the optimal linear system converge to the mirror image of the NMP zeros. An expression of this property is that the zero dynamics are "as stable in the closed loop as they are unstable in the open loop." Using $V_{0}(z)$ as a Lyapunov function we reveal an analogous property of the nonlinear zero dynamics.

Corollary (Mirroring Property): For the open- and closed-loop zero dynamics, $V_{0}(z)$ satisfies

$$
\frac{\partial V_{0}}{\partial z} f_{0}(z)=-\frac{\partial V_{0}}{\partial z}\left[f_{0}(z)+g_{0}(z) \alpha_{0}(z)\right] .
$$

This identity is immediate from (24) and (30).

\section{Example}

The essentially nonlinear character of the obtained results will be illustrated on the system

$$
\begin{aligned}
& \dot{y}=6 z^{5}+u \\
& \dot{z}=z^{3}+y .
\end{aligned}
$$

Its linearization $\dot{y}=u, \dot{z}=y$ is not detectable so that the linear analysis does not apply.

Upon the substitution of $V=V_{0}+\varepsilon V_{1}+O\left(\varepsilon^{2}\right)$ in the HJBE (19), we obtain

$$
\frac{\partial V_{0}}{\partial z}\left[z^{3}+y\right]+\frac{1}{2} y^{2}-\frac{1}{2}\left[\frac{\partial V_{1}}{\partial y}\right]^{2}+O(\varepsilon)=0 .
$$

The HJBE for the minimum energy stabilization of the zero-dynamics subsystem $\dot{z}=z^{3}+y$ is

$$
\frac{\partial V_{0}}{\partial z} z^{3}-\frac{1}{2}\left[\frac{\partial V_{0}}{\partial z}\right]^{2}=0, \quad V_{0}(0)=0 .
$$

The optimal value $V_{0}(z)$ and the corresponding control law are

$$
V_{0}(z)=\frac{z^{4}}{2}, \quad y=\alpha_{0}(z)=-\frac{\partial V_{0}}{\partial z}=-2 z^{3} .
$$

The substitution of $V_{0}(z)=z^{4} / 2$ and $\varepsilon=0$ in (40) yields $\partial V_{1} / \partial y=y+2 z^{3}$, and, hence

$$
u=-\frac{1}{\varepsilon^{2}} \frac{\partial\left(V_{0}+\varepsilon V_{1}\right)}{\partial y}=-\frac{1}{\varepsilon} \frac{\partial V_{1}}{\partial y}=-\frac{1}{\varepsilon}\left(y+2 z^{3}\right)=-\frac{1}{\varepsilon} \eta .
$$

When this control is applied to (39), the closed-loop system is

$$
\dot{\eta}=-\frac{1}{\varepsilon} \eta+6 z^{2} \eta, \quad \dot{z}=-z^{3}+\eta .
$$

Our proof of asymptotic stability of (29) was in two steps: we first showed semiglobal boundedness and then local asymptotic stability. However, for (42) we give a direct proof using $W=\eta^{2} / 2+z^{4} / 2$ as a Lyapunov function. Its derivative along the solutions of (42)

$$
\dot{W}=-\left[\begin{array}{c}
\eta \\
z^{3}
\end{array}\right]^{T}\left[\begin{array}{cc}
\frac{1}{\varepsilon}-6 z^{2} & -1 \\
-1 & 2
\end{array}\right]\left[\begin{array}{c}
\eta \\
z^{3}
\end{array}\right]
$$

is negative definite if $12 z^{2}<(2-\varepsilon) / \varepsilon$. For $\varepsilon<1$ this inequality is satisfied if $z^{2} \leq(12 \varepsilon)^{-1}$. Therefore, the invariant set $\{(\eta, z)$ : $\left.\eta^{2}+z^{4} \leq(12 \varepsilon)^{-2}\right\}$ belongs to the basin of attraction of the equilibrium $(\eta, z)=(0,0)$ of $(42)$. We conclude that the lowest achievable $L_{2}$-norm of $y$ in (39) is $J_{0}^{*}=V_{0}(z)=z^{4} / 2$. 


\section{CONClusion}

The main result in this paper establishes that the ideal performance of a nonlinear system, that is the lowest attainable $L_{2}$ norm of its output, is the least amount of energy required to stabilize the zero dynamics. Thus, unstable zero dynamics represent a structural obstacle to attaining zero ideal performance. Although our results are for systems of relative degree one with a local growth condition, a similar structural obstacle is present in a wider class of nonlinear systems for which its quantification would require a more elaborate analysis.

\section{ACKNOWLEDGMENT}

The authors are grateful to J. Grizzle for a critical remark and to A. Teel for many helpful suggestions.

\section{REFERENCES}

[1] M. M. Seron, J. H. Braslavsky, and G. C. Goodwin, Fundamental Limitations in Filtering and Control. New York: Springer, 1997.

[2] H. Kwakernaak and R. Sivan, Linear Optimal Control Systems. New York: Wiley, 1972.

[3] L. Qiu and E. J. Davison, "Performance limitations of nonminimum phase systems in the servomechanism problem," Automatica, vol. 29, no. 2, pp. 337-349, 1993.

[4] R. H. Middleton, "Trade-offs in linear control systems design," Automatica, vol. 27, no. 2, pp. 281-292, Mar. 1991.

[5] A. Jameson and R. E. O'Malley, Jr., "Cheap control of the time-invariant regulator," Appl. Math. Optim., vol. 1, no. 4, pp. 337-354, 1975.

[6] P. V. Kokotović, H. K. Khalil, and J. O'Reilly, Singular Perturbation Methods in Control: Analysis and Design. New York: Academic, 1986.

[7] J. S. Freudenberg and D. P. Looze, "Right half plane poles and zeros and design tradeoffs in feedback systems," IEEE Trans. Automat. Contr., vol. 30, pp. 555-565, June 1985.

[8] M. M. Seron, J. H. Braslavsky, P. V. Kokotović, and D. Q. Mayne, "Feedback limitations in nonlinear systems: From Bode integrals to cheap control," CCEC, University of California, Santa Barbara, CA, Tech. Rep. 97-0304, 1997. Available at http://www-ccec.ece.ucsb. edu/techrpts/index.html\#1997.

[9] J. H. Braslavsky, M. M. Seron, D. Q. Mayne, and P. V. Kokotović, "Limiting performance of optimal linear filters," Automatica, vol. 35, no. 2, pp. 189-199, Feb. 1999.

[10] A. Isidori, Nonlinear Control Systems, 3rd ed. New York: Springer, 1995.

[11] R. Sepulchre, M. Janković, and P. V. Kokotović, Constructive Nonlinear Control. New York: Springer, 1997.

[12] P. D. Christofides and A. R. Teel, "Singular perturbations and input-tostate stability," IEEE Trans. Automat. Contr., vol. 41, pp. 1645-1650, Nov. 1996

[13] A. Saberi and H. Khalil, "Quadratic-type Lyapunov functions for singularly perturbed systems," IEEE Trans. Automat. Contr., vol. 29, pp. 542-550, June 1984.
A New Representation of the Parameters of Lifted Systems

\author{
Leonid Mirkin and Zalman J. Palmor
}

\begin{abstract}
Lifting, i.e., discretization with built-in intersample behavior, is an emerging technique for the analysis and design of sampled-data systems. The applicability of the lifting technique, however, is severely limited owing to difficulties in dealing with the parameters of the lifted systems, which are operators over infinite-dimensional spaces rather than finite-dimensional matrices. In this paper, a new representation for the parameters of the lifted systems is proposed. The technical machinery developed in the paper based on this representation simplifies considerably algebraic manipulation over parameters of the lifted systems, thus extending the scope of applicability of the lifting technique. To illustrate the advantages of the proposed approach, the computational issues in the sampled-data $H^{\infty}$ problem are considered.
\end{abstract}

Index Terms - Generalized sampling and hold circuits, lifting technique, sampled-data systems.

\section{INTRODUCTION}

The main motivation for this paper comes from the consideration of the intersample behavior of sampled-data systems, that is systems consisting of a continuous-time plant and a discrete-time controller, which are connected by sampling and hold devices. Dealing with the continuous-time behavior of sampled-data systems is complicated by 1) the simultaneous presence of continuous- and discrete-time signals and 2) inherent periodicity of the interconnection of a sampler, a discrete-time controller, and a hold. An elegant way to circumvent these difficulties is offered by the so-called lifting technique [1]-[3] (see also [4] and [5]), which allows one to convert periodic continuous/discrete systems to equivalent, in some sense, pure discrete-time shift-invariant ones.

Although the reduction of hybrid periodic problems to discretetime time-invariant ones is clearly advantageous, the lifting method gives rise to another problem: input and output spaces of the lifted systems become infinite-dimensional. Conceptually, this fact does not lead to considerable difficulties since many LTI system notions have almost one-to-one counterparts in the lifted domain (see [3] and [6]). Moreover, the preservation of the state-space dimensions under lifting guarantees that any observer-based (including $H^{2}$ and $H^{\infty}$ ) controller in the lifted domain has a finite state dimension. Thus, analytic solutions to various sampled-data analysis and design problems can be obtained in terms of the parameters of the lifted models in quite a straightforward manner. Yet the latter parameters are no longer finite-dimensional matrices but rather operators over infinite-dimensional input and/or output spaces, such as $L^{2}[0, h]$. For this reason, the usefulness of a lifted solution relies essentially upon the ability to treat such infinite-dimensional operators.

Unfortunately, lifted parameters are not readily treatable. Consequently, sampled-data problems solved so far by means of the lifting technique share one key property: the possibility to separate beforehand the infinite dimensionality from the design by converting the problem to an equivalent finite-dimensional one. If a problem admits such a separation, the complexity of the operations over the

Manuscript received December 22, 1995; revised March 5, 1997 and February 4, 1998. Recommended by Associate Editor, M. Araki. The work of L. Mirkin was supported by the Center for Absorption in Science, Ministry of Immigrant Absorption, State of Israel.

The authors are with the Faculty of Mechanical Engineering, Technion-IIT, Haifa 32000, Israel.

Publisher Item Identifier S 0018-9286(99)02107-8. 